\title{
Expression of A disintegrin and metalloprotease 10 in pancreatic carcinoma
}

\author{
MATTHIAS M. GAIDA ${ }^{1 *}$, NATASCHA HAAG ${ }^{2 *}$, FRANK GÜNTHER ${ }^{2}$, DARJUS F. TSCHAHARGANEH ${ }^{1}$,

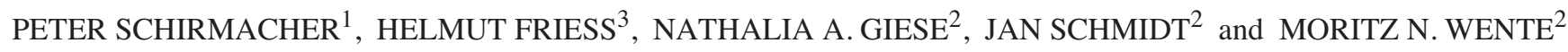 \\ ${ }^{1}$ Institute of Pathology; ${ }^{2}$ Department of Surgery, University of Heidelberg, Heidelberg; \\ ${ }^{3}$ Department of Surgery, Technische Universität München, Munich, Germany
}

Received March 16, 2010; Accepted April 28, 2010

DOI: 10.3892/ijmm_00000463

\begin{abstract}
The protease ADAM10 influences progression and metastasis of cancer cells and is overexpressed in various malignancies. Therefore, the aim of our study was to evaluate the expression and potential function of ADAM10 in the pathophysiology of pancreatic cancer (PDAC). ADAM10 expression in normal pancreatic (NP), chronic pancreatitis (CP), PDAC tissues, as well as PDAC cell lines was determined. To evaluate whether rhADAM10 or ADAM10 silencing influences cancer cell viability, MTT assay was used. Matrigel invasion and wound healing assays were performed to observe influence on invasion and migration. ADAM10 mRNA was expressed in all samples of NP, CP and PDAC tissue and cell lines. Western blotting and immunohistochemistry revealed stronger ADAM10 expression in PDAC than in NP. ADAM10 silencing or rhADAM10 had no effect on cell viability. ADAM10 silencing markedly reduced invasiveness and migration of cancer cells. These findings establish ADAM10 as a contributing factor in PDAC invasion and metastasis.
\end{abstract}

\section{Introduction}

Pancreatic ductal adenocarcinoma (PDAC) is the fourth leading cause of cancer death with an incidence that equals almost its prevalence (1). Aggressive growth behavior, high rate of invasiveness, early metastasis, and resistance to radiation and chemotherapy are factors determining the dismal prognosis of this disease, with an overall 5-year survival rate of $<5 \%$ (1).

Recently, it has been described that the family of A disintegrin and metalloprotease (ADAMs) can influence the

Correspondence to: Dr Moritz N. Wente, Department of Surgery, University of Heidelberg, Im Neuenheimer Feld 110, D-69120 Heidelberg, Germany

E-mail: moritz.wente@med.uni-heidelberg.de; m.wente@web.de

*Contributed equally

Key words: A disintegrin and metalloprotease 10, pancreatic carcinoma, invasiveness, migration invasiveness and tumor cell dissemination of PDAC $(2,3)$. ADAMs belong to the Type I transmembrane glycoproteins containing metalloproteinase and disintegrin domains (4). They play pivotal roles in cell adhesion and function via fusion with the disintegrin domain, intracellular signaling through the cytoplasmatic domain and in proteolysis of membrane proteins, also defined as ectodomain shedding (5-7).

ADAMs possess the ability to regulate a variety of physiological functions for example in the fertilization process $(8,9)$, myogenesis $(10)$, adipogenesis $(11,12)$, and neurogenesis (13). They are involved in disease processes, via their protease activity, such as inflammation, asthma, dementia and cancer (14-16). ADAM10 was originally isolated as a myelin basic protein-degrading enzyme from bovine brain encoding a protein of 748 amino acids (17) with a molecular weight of $\sim 100 \mathrm{kDa}$ as unprocessed proform and $\sim 60 \mathrm{kDa}$ as mature, enzymatic active form (18). Substrates shedded by ADAM10 comprise cytokines such as TNF- $\alpha(19,20)$, CX3CL1 $(21,22)$ and CXCL16 (22-24), as well as the adhesion molecules L1-CAM (25), N-cadherin (26), and E-cadherin (27). As described for various members of the ADAM family (16), ADAM10 has been reported to be overexpressed in human malignancies, e.g. oral squamous cell carcinoma where it appears to promote growth (28). Furthermore, McCulloch et al showed the expression of ADAM10 and its regulation by dihydrotestosterone, insulin-like growth factor I, and EGF in prostate cancer (18).

An enhancement of tumor cell dissemination in ovarian and uterine carcinomas (29) as well as an increased invasiveness of colon carcinoma (30) mediated by ADAM10 has been described in previous studies. The loss of the cell adhesion molecule E-cadherin, a main substrate of ADAM10, was shown to promote invasiveness of tumors (31-33). Recently, the expression of four members of the ADAM family, ADAM8 (3), ADAM9 (34), ADAM15 (35), and ADAM17 (2), has been evaluated in PDAC. The fact that low ADAM8 expression correlates with longer median survival of patients suffering from PDAC implicates a high biological value of ADAMs in pancreatic cancer pathogenesis (3). The role of ADAM10 in pancreatic disease remains unknown. Therefore, the aim of our study was to evaluate the expression and potential function of ADAM10 in the pathophysiology of PDAC, focused on the ability to promote pancreatic cancer cell invasion and migration. 


\section{Materials and methods}

Tissue collection in patients with pancreatic carcinoma. Tissue samples of PDAC or CP were obtained upon informed consent from patients undergoing pancreatic resection at the Department of Surgery, University of Heidelberg, Germany. Pancreatic tissues from healthy donors were collected through an organ donor program when there was no suitable recipient. The histological diagnosis of all samples was evaluated by an experienced pathologist.

For immunohistochemical procedures freshly removed tissue samples were fixed in $4 \%$ formaldehyde solution for $24 \mathrm{~h}$ and embedded in paraffin. In addition, samples were frozen in liquid nitrogen immediately after surgical removal and maintained at $-80^{\circ} \mathrm{C}$ until use or preserved in RNAlater (Ambion Europe, Huntington, UK). The ethics committee of the University of Heidelberg approved experiments with the collected samples.

Immunohistochemistry. Immunohistochemical staining was performed on $3 \mu \mathrm{m}$ thick paraffin-embedded tissue sections from PDAC $(n=15), C P(n=7)$ and healthy donors $(n=5)$. After deparaffinization in xylene and rehydration in graded alcohols, antigen retrieval by microwave treatment for $15 \mathrm{~min}$ in citrate buffer ( $\mathrm{pH}$ 6.0) was performed. Endogenous peroxidase activity was blocked with $0.3 \%$ hydrogen peroxidase for $10 \mathrm{~min}$. Then, sections were incubated for $30 \mathrm{~min}$ at room temperature (RT) with normal goat serum prior to overnight incubation at $4^{\circ} \mathrm{C}$ with a polyclonal rabbit anti-human-ADAM10 (1:200) antibody (Chemicon, Schwalbach, Germany). For negative control, ADAM10 primary antibody was pre-absorbed with a purified blocking peptide (Chemicon) to exclude nonspecific binding. The following day, after washing 3 times with TBS/0.1\% BSA$0.5 \%$ Tween-20, slides were incubated with a goat anti-rabbit antibody solution (Dako Cytomation, Hamburg, Germany) for 45 min at RT. For visualization antibody-binding DAB+ Chromogen (Dako Cytomation) was used according to the manufacturer's protocol. Sections were counterstained with Mayer's hemalaun, rehydrated with graded ethanol, mounted, and analyzed by standard light microscopy. For semiquantitative analyses slides were scored by two observers independently. First ADAM10 staining pattern of different structures was analyzed and subsequently its intensity was categorized as focal, diffuse or negative.

Cell lines and culture conditions. The human PDAC cell lines ASPC-1, BxPc-3, Capan-1, MiaPaCa-2 and Su8686 were purchased from American Type Culture Collection (ATCC, Rockville, MD, USA); COLO-357 and T3M4 were a gift from R. Metzgar (Duke University, Durham, NC, USA). All cell lines were cultured in RPMI-1640 medium containing 10\% fetal bovine serum (FBS), penicillin $(100 \mathrm{U} / \mathrm{ml})$ and streptomycin $(100 \mu \mathrm{g} / \mathrm{ml})$ (Invitrogen, Karlsruhe, Germany). Cells were incubated at $37^{\circ} \mathrm{C}$ in humidified air with $5 \% \mathrm{CO}_{2}$.

Quantitative real-time polymerase chain reaction ( $q R T-P C R$ ) analysis. mRNA and cDNA preparation kits were purchased from Roche Applied Sciences (Mannheim, Germany). For mRNA preparation, we used the automated MagNA Pure LC instrument and corresponding isolation kit I (for cells) and kit II (for tissue samples). Primers were obtained from Search-LC (Heidelberg, Germany). cDNA for RT-PCR was prepared by using a first strand cDNA Synthesis kit. Subsequently, RT-PCR was performed with the LightCycler-FastStart DNA SYBR Green kit. Cyclophilin-B (CPB) was used as a housekeeping gene to normalize the expression of specific ADAM10 transcripts and presented as adjusted copies/10 k copies CPB, as previously described (36).

Western blotting. Total protein was isolated from tissues and cell lines (ASPC-1, BxPc-3, Capan-1, COLO-357, MiaPaCa-2, Su-8686, Jurkat) and ADAM10 siRNA transfected cells (ASPC-1, COLO-357). Protein concentration was measured using the micro-BCA protein assay (Perbio, Bonn, Germany). Proteins $(25 \mu \mathrm{g})$ were denatured with NuPAGE 4x LDS Sample Buffer (Invitrogen) and NuPAGE Sample Reducing Agent (10x) (Invitrogen) at $70^{\circ} \mathrm{C}$ for $10 \mathrm{~min}$. Protein lysates of Jurkat cells served as positive control for the ADAM10 antibody and ZR-75-1 cell lysate (Santa Cruz, Heidelberg, Germany) for E-cadherin antibodies. Tissue and cellular protein lysates were separated by electrophoresis in 10-12\% Bis-Tris-gels (Invitrogen), transferred to nitrocellulose membranes and blocked for $1 \mathrm{~h}$ at RT with $5 \%$ non-fat dry milk in TTBS (20 mM Tris- $\mathrm{HCl}, 150 \mathrm{mM} \mathrm{NaCl}$, and $0.1 \%$ Tween-20; 5\% M-TTBS). The membranes were incubated with primary antibodies, rabbit anti-human-ADAM10 (1:600) (Chemicon), monoclonal mouse anti-human E-cadherin (C-Terminus, BD Biosciences, Heidelberg, Germany; 1:1,000), and polyclonal rabbit anti-human E-cadherin (N-Terminus, Santa Cruz; $1: 1,000)$ diluted in $5 \%$ M-TTBS at $4^{\circ} \mathrm{C}$ overnight. Membranes were washed with TTBS and blocked for $1 \mathrm{~h}$ at RT with 5\% M-TTBS. After blocking, membranes were exposed to the secondary antibody HRP-conjugated antirabbit (Amersham Life Science, Amersham, UK) or HRPconjugated anti-mouse (Santa Cruz, Biotechnologies, Santa Cruz, CA, USA) in a dilution of 1:2,500 in 5\% M-TTBS for $1 \mathrm{~h}$ at RT. Signals were detected using an enhanced chemoluminescence reaction kit (ECL Western blotting detection (Amersham). To assess the equivalence of sample loading Simply Blue Safe Stain (Invitrogen) was used.

Cell proliferation assay using the 3-(4,5-Dimethylthiazol-2yl)-2,5-diphenyltetrazolium bromide (MTT) dye method. ASPC-1 and COLO-357 cells were seeded into a 96-well tissue culture plate at an initial number of $5 \times 10^{3}$ cells/well in $100 \mu 1$ of complete medium. Cells were allowed to attach overnight. The next day, $100 \mu 1$ RPMI, containing different concentrations of enzymatic active rhADAM10 with final concentrations of $5,10,50$ and $100 \mathrm{ng} / \mathrm{ml}$ or control were added.

After an incubation time of 48 or $72 \mathrm{~h}$, MTT was added to a final concentration of $0.5 \mathrm{mg} / \mathrm{ml}$ and incubated at $37^{\circ} \mathrm{C}$ and $5 \% \mathrm{CO}_{2}$ for $4 \mathrm{~h}$. Then supernatants were aspirated, plates were dried at RT for $3 \mathrm{~h}$ and finally $100 \mu \mathrm{l}$ of acidic isopropanol was added to each well. The optical density (OD) was measured at $570 \mathrm{~nm}$ to compare treatment and control groups. All experiments were done at least thrice in triplicates.

siRNA transfection for silencing ADAM10. ASPC-1 and COLO-357 cells were grown to $50-70 \%$ confluence in a 
6-well plate before they were transfected with two different specific ADAM10 siRNAs, siRNA1 (Ambion Applied Biosystems, Darmstadt, Germany) and siRNA2 (Qiagen, Hilden Germany). Non-targeting scrambled siRNA (negative control siRNA, Qiagen) served as negative control. Cells were transfected using $15 \mu \mathrm{l}$ of transfection reagent (RNAifect Transfection Reagent, Qiagen) according to the manufacturer's instructions.

The sequences were, siRNA1 sense GGAUAACAGAGA AUGGUGGtt, siRNA1 antisense CCACCAUUCUCUGUU AUCCtg; siRNA2 sense CAAACUUCACAGACUGUAAtt; siRNA2 antisense UUACAGUCUGUGAAGUUUGgt; control siRNA sense UUCUCCGAACGUGUCACGUtt, control siRNA antisense ACGUGACACGUUCGGAGAAtt. The siRNA and the transfection reagent were preincubated in ECR-buffer (Qiagen) (100 $\mu \mathrm{l}$ containing $5 \mu \mathrm{g}$ ADAM10 siRNA and $5 \mu \mathrm{g}$ control siRNA) at RT for $15 \mathrm{~min}$, before the mixture was added to each well. After $24 \mathrm{~h}$, medium was replaced by serum-free medium and cells were incubated for another $24 \mathrm{~h}$ at $37^{\circ} \mathrm{C}$ and $5 \% \mathrm{CO}_{2}$. Subsequently, efficiency of mRNA knockdown was measured $48 \mathrm{~h}$ after transfection by qRT-PCR and at the protein level by Western blotting.

Invasion assay. In vitro invasiveness of the ADAM10-siRNA transfected cell lines ASPC-1 and COLO-357 was determined by using Biocoat Matrigel Invasion Chambers (BD Biosciences, Heidelberg, Germany), consisting of a 24-well plate and Matrigel-precoated cell culture inserts with an $8-\mu \mathrm{m}$ pore size. Mock-transfected cells served as control. First, the Matrigel membrane was rehydrated with $500 \mu 1$ of serumfree RPMI-medium by incubating the plate at $37^{\circ} \mathrm{C}$ and $5 \%$ $\mathrm{CO}_{2}$ for $2 \mathrm{~h}$. Then, $500 \mu \mathrm{l}$ of each cell suspension ASPC-1 $\left(1.5 \times 10^{5}\right.$ cells $\left./ \mathrm{ml}\right)$ and COLO-357 $\left(3 \times 10^{5}\right.$ cells $\left./ \mathrm{ml}\right)$ in RPMImedium containing $1 \%$ FCS was seeded onto the upper surface of the cell culture inserts. RPMI-medium containing $10 \%$ FCS was added into the lower chamber. The assay was incubated at $37^{\circ} \mathrm{C}$ in a $5 \% \mathrm{CO}_{2}$ humidified atmosphere for $48 \mathrm{~h}$. After incubation, non-invading cells were removed completely from the membrane by using a cotton-tipped swab. The membranes were fixed in ice-cold methanol for $20 \mathrm{~min}$ and invading cells were stained with $1 \%$ toluidine blue (Sigma-Aldrich, Taufkirchen, Germany). Invading cells that adhered to the lower surface of the insert were quantified by a light microscope counting the complete membrane cutout (membrane surface area $0.3 \mathrm{~cm}^{2}$ ). The assay was performed in duplicate and repeated three times.

In vitro wound healing. ASPC-1 and COLO-357 cells were seeded in 6-well plates, transfected with scrambled siRNA or specific ADAM10 siRNA and cultured until they reached confluence. A cell-free area was introduced by scraping the monolayer with a pipette tip (20 $\mu$ 1, Sarstedt, Nümbrecht, Germany). Cells were washed with PBS and $2 \mathrm{ml}$ medium containing $1 \%$ FCS was added. To monitor wound closure, cells were photographed at baseline and at defined time periods under standard culture conditions, respectively (Leica DFC300 FX, Wetzlar, Germany). To quantify the results, pictures were analyzed by an Image Analysis System (Zeiss Axiovision Release 4.2, Jena, Germany). Relative migratory activity was determined by calculating cell-free areas.
Statistical analysis. Statistical analysis was performed by using Prism4 software (GraphPad, San Diego, CA). The Mann-Whitney U test or analysis of variance, followed by Dunnett's multiple comparison tests were performed where appropriate. Significance was defined as $\mathrm{p}<0.05$.

\section{Results}

Expression of ADAM10 in pancreatic cancer cell lines and pancreatic tissue samples. To analyze ADAM10 tissue expression at the protein level immunoblot analysis was carried out. In 6 of 7 tested cell lines the $\sim 100$-kDa proform of ADAM10 and in 3 of 7 cell lines the $\sim 60-\mathrm{kDa}$ active, processed form was detectable on a protein level. Overall, there was a heterogeneous expression of ADAM10 in the tested cell lines e.g. ASPC-1 cells showed only weak expression of ADAM10, while COLO-357 cells revealed a very strong expression (Fig. 1A). QRT-PCR was performed to evaluate the expression of ADAM10 mRNA in pancreatic cancer cell lines $(n=7)$. ADAM10 mRNA was detected in all PDAC cell lines with markedly high ADAM10 copy numbers varying between 4,368 \pm 30 copies ASPC- 1 and 1,397 \pm 223 copies/10 k copies CPB in COLO-357, respectively (Fig. 1B).

We performed qRT-PCR to compare the in vivo expression pattern of ADAM10 in normal and diseased pancreas using RNA isolated from normal donor tissue $(n=25), C P(n=55)$ and PDAC $(n=79)$. The analysis of tissue samples revealed that the median of ADAM10 mRNA levels were not significantly higher in PDAC with 268 copies compared to NP tissue with 275 copies, but significantly higher $(\mathrm{P}<0.01)$ compared to $\mathrm{CP}$ with 164 copies/10 k copies CPB, respectively (Fig. 1C). At the protein level, in contrast to the qRT-PCR data, ADAM10 was only weakly expressed in normal tissue as an $\sim 60$ - and $\sim 100$-kDa protein. A stronger signal of ADAM10 protein was detected in $\mathrm{CP}$ and PDAC compared to normal tissue, without a distinct difference between PDAC and CP (Fig. 1D). There was a difference in the expression of the proform and mature form between cell lysates and tissue samples. In cell lysates predominantly the proform was detectable whereas in tissue samples a stronger expression of the active form was observed. In addition to the $\sim 60-\mathrm{kDa}$ processed form and the 100-kDa unprocessed form of ADAM10 an additional band with a molecular weight of $\sim 55 \mathrm{kDa}$ was detected in tissue samples but not in cell lysates. This band might represent a splice variant or a degradated protein form of ADAM10 (37). For determination of the exact localization of ADAM10 in normal pancreatic tissue, $\mathrm{CP}$ and PDAC, immunohistochemistry was carried out. Immunoreactivity revealed differences between normal pancreatic tissue, $\mathrm{CP}$ and PDAC (Fig. 1E).

A semiquantitative analysis of the immunohistochemistry results revealed the following results. In NP tissues $(n=5)$, exocrine cells and islets showed a weak, diffuse reactivity for ADAM10; a focal expression of ADAM10 was observed in normal ducts and vessels; positive immunoreactivity for ADAM10 was also detected in nerves and stroma. In CP specimens $(n=7)$ ADAM10 exhibited intense immunostaining particularly in tubular complexes, inflammatory cells showed a focal reactivity for ADAM10. In all analyzed pancreatic cancer tissues $(n=15)$, tumor cells revealed a positive staining 
A

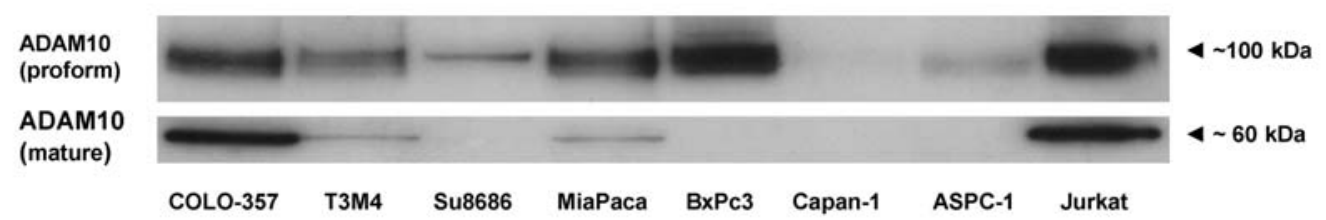

B
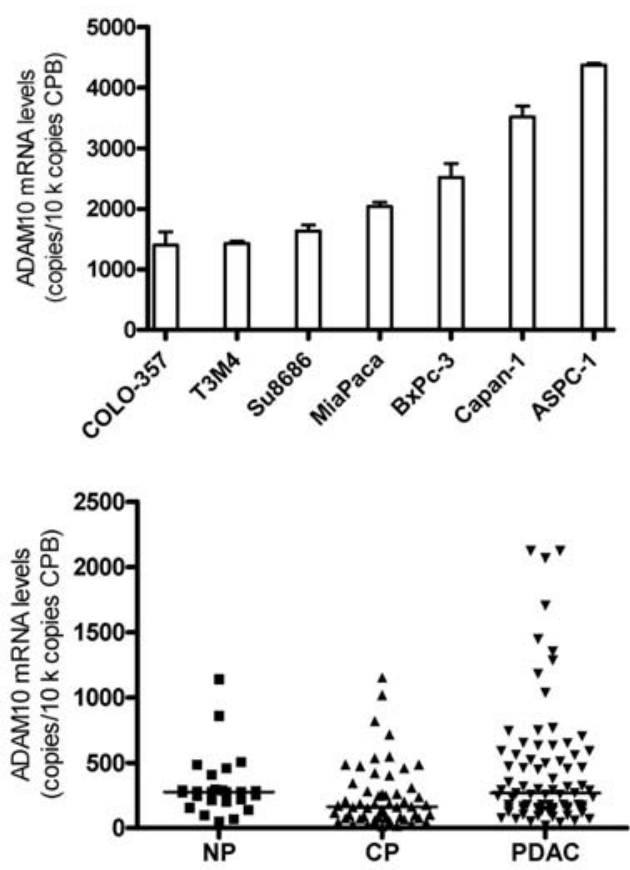

D

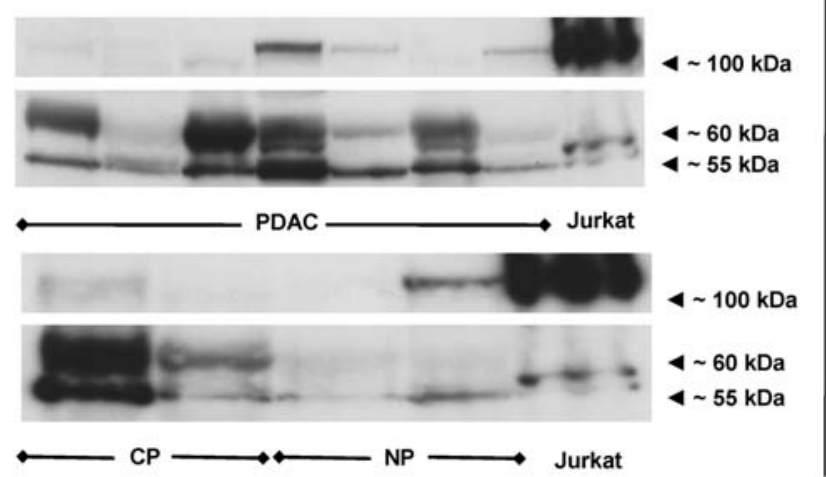

E
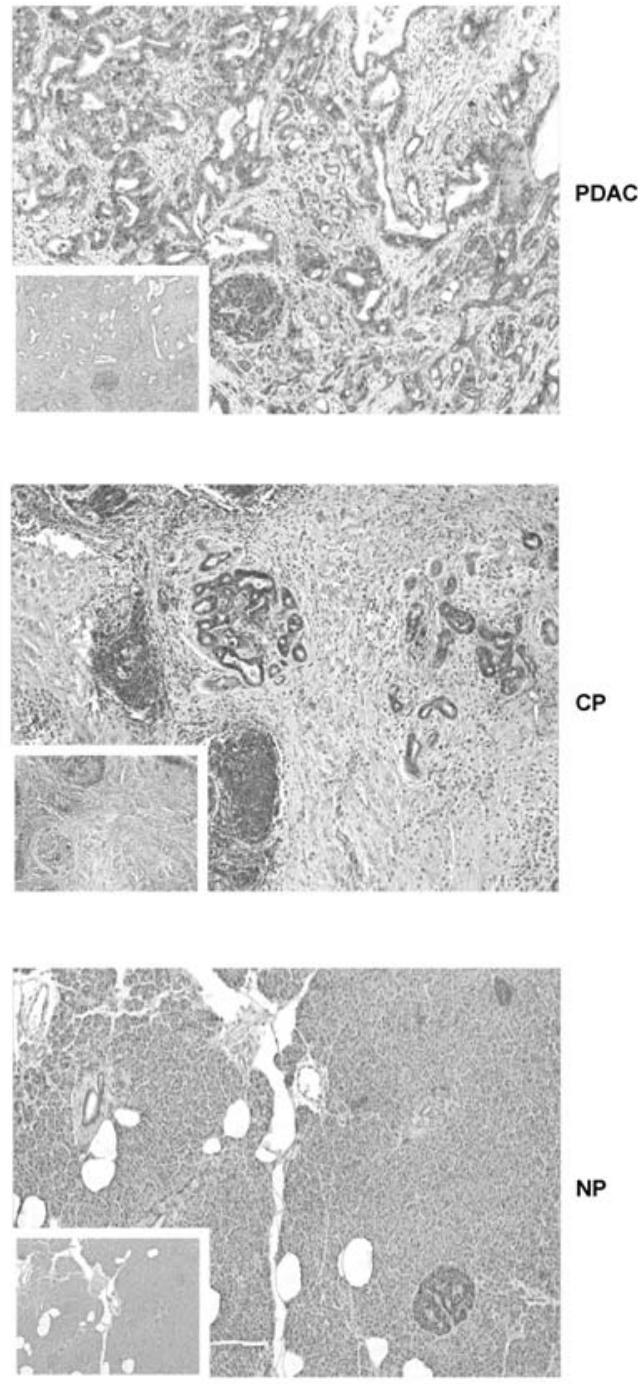

Figure 1. Expression of ADAM10 in pancreatic cancer cell lines and pancreatic tissue samples. A. Expression of ADAM10 protein in pancreatic cancer cells, 6 of 7 tested cell lines displayed protein expression for ADAM10. Jurkat cells served as a positive control. B. Expression of ADAM10 mRNA in pancreatic cancer (PDAC) cells. All tested cell lines displayed ADAM10 mRNA expression. C. Expression of ADAM10 mRNA in pancreatic tissues (qRT-PCR). ADAM10 mRNA expression levels in normal pancreas NP) $(n=25)$, chronic pancreatitis $(C P)(n=55)$ and pancreatic cance (PDAC) tissue (n=79). D. Expression of ADAM10 in pancreatic tissues. ADAM10 protein expression in NP $(n=2), C P(n=2)$ and PDAC $(n=7)$ tissue. Jurkat cells served as positive control. In contrast with the results of the qRT-PCR, there was a trend toward upregulation of ADAM10 protein in CP as well as in PDAC compared to NP. E. Expression and localization of ADAM10 in pancreatic tissues based on immunohistochemistry. Representative ADAM10 immunoreactions in NP, CP and PDAC are shown. The lack of staining in consecutive negative control tissue sections is shown as inserts.

for ADAM10 with staining mainly concentrated in the cytoplasm, but it was also recognizable in the nucleus. Nonspecific binding of ADAM10 antibody was not detectable after preincubation with the specific blocking peptide.

Effects of rhADAM10 and ADAM10-specific siRNA transfection on cell growth. A crucial role of ADAMs is the shedding of growth factors such as TGF- $\alpha$ and HB-EGF, and this processing may alter signaling on the surfaces of cancer cells and result in enhanced cell proliferation by activating autocrine and paracrine mechanisms (38-41). ADAM10 also contributes to cell proliferation by modulating B-catenin signaling through E-cadherin shedding and increasing cyclin D1 levels $(27,42)$. In human mesangial cells ADAM10 inhibition led to a strong reduction of cell proliferation (43). To investigate the influence of ADAM10 on PDAC cell viability, ASPC-1 and COLO-357 cells were incubated with enzymatically active rhADAM10, followed by MTT assays. No significant effects of rhADAM10 on cell viability in the tested cell lines up to a concentration of $100 \mathrm{ng} / \mathrm{ml} \mathrm{rhADAM} 10$ 

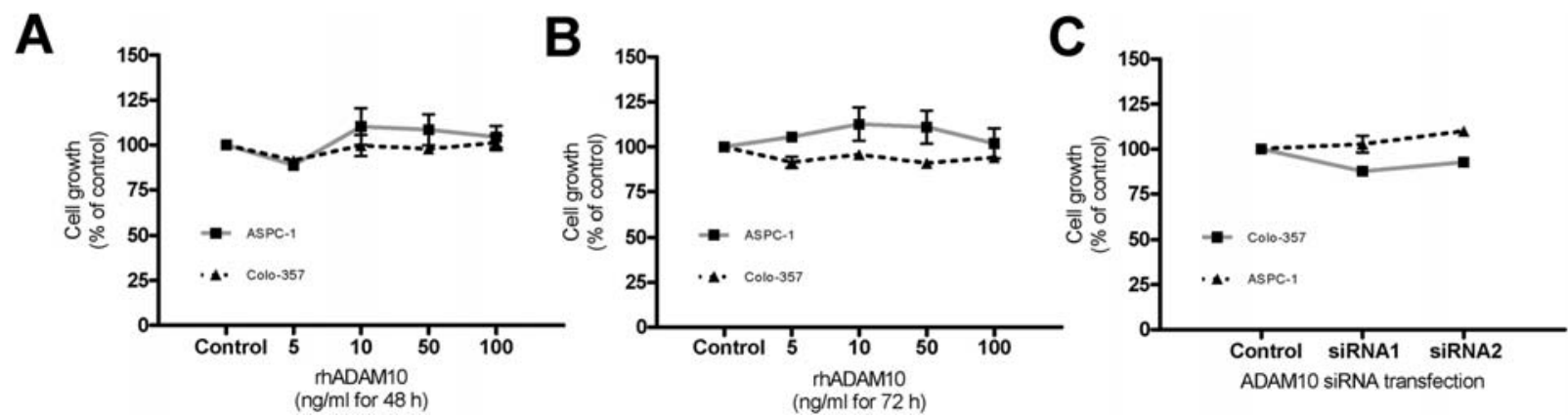

Figure 2. Effects of rhADAM10 and ADAM10-specific siRNA transfection on cell growth. A-C. Effect of rhADAM10 and ADAM10 gene silencing by using siRNA on PDAC cell growth. The indicated pancreatic cancer cell lines were treated with 5, 10, 50 and $100 \mathrm{ng} / \mathrm{ml}$ rhADAM10. A, B. MTT assays were performed after incubation for 48 and $72 \mathrm{~h}$, respectively. There was no significant effect of rhADAM10 on cell growth detectable. C. PDAC cells were transfected with ADAM10 siRNA. MTT assays were performed after incubation for $48 \mathrm{~h}$. Both siRNA oligonucleotides did not significantly influence cell growth. Data are presented as mean \pm SEM of three independent experiments.

A
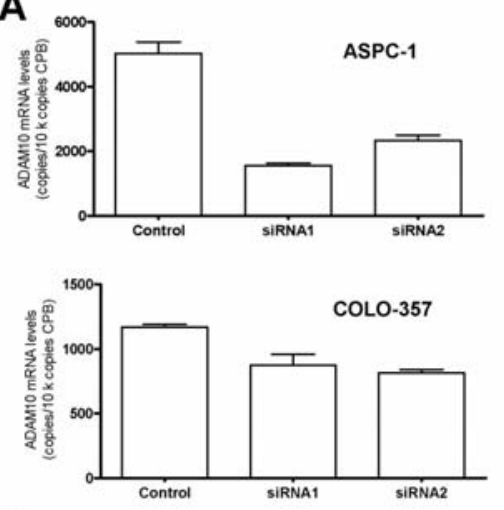

D

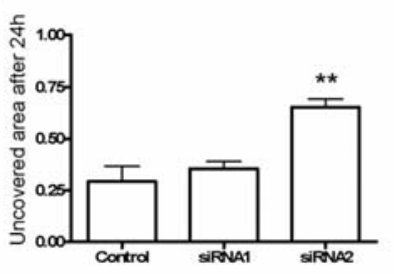

E

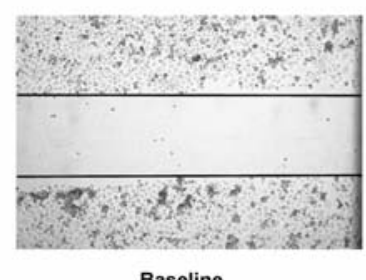

Baseline

B

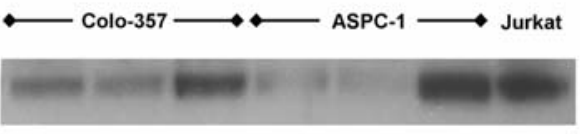

ADAM10

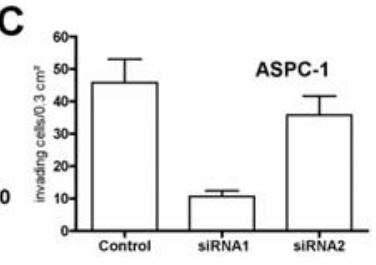

SiRNA2 siRNA1 control siRNA2 siRNA1 control
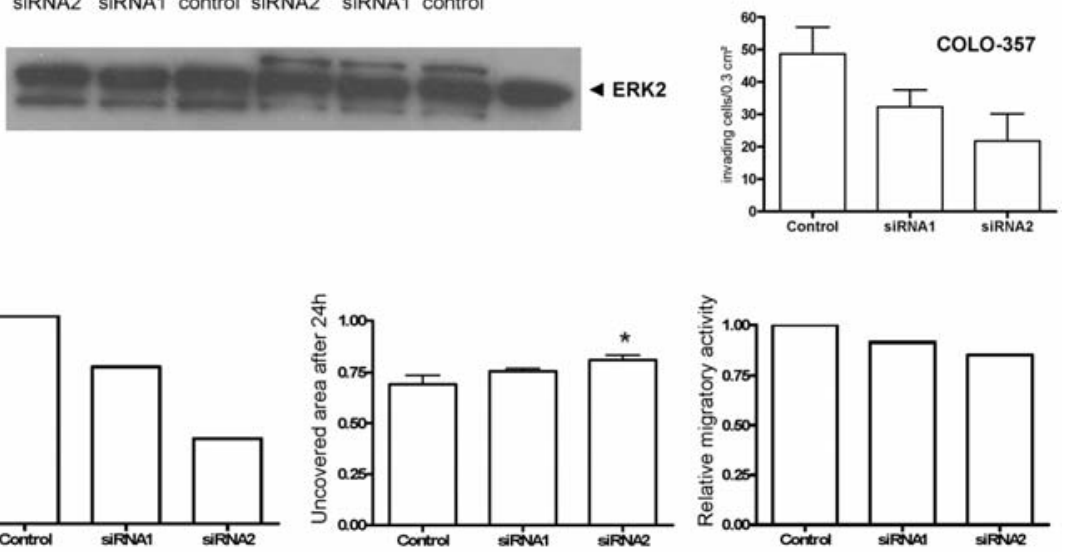

COLO-357

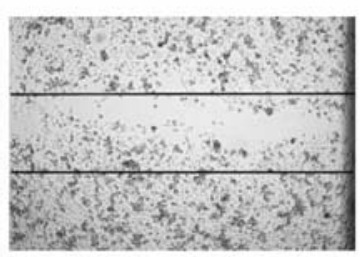

24h siRNA1

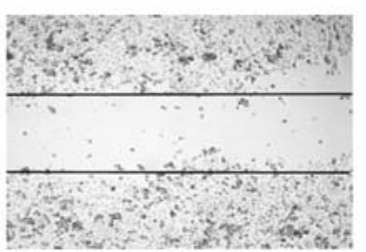

24h SIRNA2

Figure 3. Effects of ADAM10 on invasiveness and migration of PDAC cells. A. Effect of ADAM10 siRNA transfection on ADAM10 mRNA level in ASPC-1 and COLO-357 cells $48 \mathrm{~h}$ after transfection. B. Effect of ADAM10 siRNA transfection on protein level in ASPC-1 and COLO-357 cells $48 \mathrm{~h}$ after transfection. Both siRNA oligonucleotides reduced ADAM10 mRNA transcripts and expression on protein level. Data are presented as mean \pm SEM of three independent experiments. C. Effect of ADAM10 gene silencing with ADAM10 siRNA on the invasiveness of ASPC-1 and COLO-357 cells as assayed by a standardized Matrigel assay. Invasiveness was markedly reduced after ADAM10 siRNA transfection for $48 \mathrm{~h}$. The membrane surface area was $0.3 \mathrm{~cm}^{2} .{ }^{*} \mathrm{p}<0.05$, ${ }^{* *} \mathrm{p}<0.01$. D. Migration of ASPC-1 and COLO-357 cells, assayed by an in vitro wound healing assay, was also markedly reduced after ADAM10 siRNA transfection. The uncovered area $\left(\right.$ pixel $\left.^{2}\right) 24 \mathrm{~h}$ after wounding was measured using an image analyzing system and the relative migratory activity was determined by normalizing to the control. Data are presented as mean \pm SEM of three independent experiments. ${ }^{*} \mathrm{p}<0.05,{ }^{* *} \mathrm{p}<0.01$. E. One representative of three independent experiments is shown. Photographs were taken from ASPC-1 cell lines at baseline and $24 \mathrm{~h}$ after transfection with scrambled (control) siRNA, siRNA1, and siRNA2, respectively.

after incubation for 48 and $72 \mathrm{~h}$ were detected (Fig. 2A and B). On the other hand, a toxic effect of rhADAM10 was also not recognized. Silencing of ADAM10 by specific siRNA transfection, which decreased mRNA levels by $69.1 \%$ (siRNA1) and $49.9 \%$ (siRNA2) in ASPC-1 cells and by $25.3 \%$ (siRNA1) and $30.2 \%$ (siRNA2) in COLO-357 cells (see below) did not significantly influence anchorage-dependent cell growth either (Fig. 2C). 
A
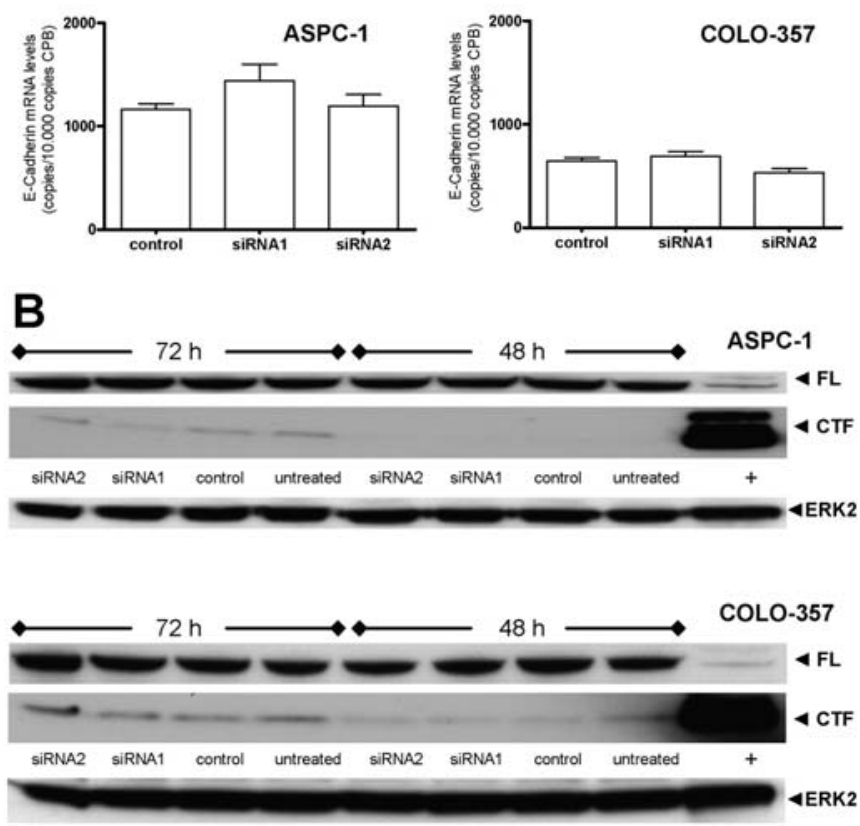

Figure 4. Effect of ADAM10 gene silencing on E-cadherin expression. A. Effect of ADAM10 siRNA transfection on mRNA E-cadherin expression. In ASPC-1 and COLO-357 cells no significant effect of ADAM10 silencing on E-cadherin expression was detectable. Data are presented as mean \pm SEM of three independent experiments. B. Effect of ADAM10 siRNA on E-cadherin protein levels. Expression on protein level was analyzed by Western blotting. In ASPC-1 and COLO-357 cells. No significant effect of ADAM10 siRNA transfection on CTF1 and FL E-cadherin expression was observable. Interestingly, the CTF1 band appeared in COLO-357 cells after $48 \mathrm{~h}$ whereas in ASPC-1 cells the band was only detectable after $72 \mathrm{~h}$.

Effects of ADAM10 on invasiveness and migration of PDAC cells. In vitro experiments adressing tumor invasiveness using Matrigel or basement membrane proteins demonstrated that invasiveness of tumor cells is decreased by metalloproteinase inhibitors $(44,45)$. ADAMs have the potential to regulate both ECM remodeling and cell migration $(46,47)$. Recently, it was shown that ADAM8 silencing significantly reduced pancreatic cancer cell invasion (3). ADAM10-mediated release enhanced tumor cell dissemination by increasing cell migration in ovarian and uterine carcinomas (29).

ASPC-1 and COLO-357 were used for siRNA transfection experiments. The $48 \mathrm{~h}$ after ADAM10 siRNA application, effectiveness of the transfection was evaluated by qRT-PCR and Western blot analysis. A significant reduction of ADAM10 expression at the mRNA level was observed in ASPC-1 and COLO-357 cells using siRNA1 or siRNA2 (Fig. 3A). Western blot analyses confirmed these results at the protein level (Fig. 3B).

To examine the influence of ADAM10 on the invasiveness of pancreatic cancer cell lines, standardized Matrigel invasion assays were used. ASPC-1 and COLO-357 cells were transfected with ADAM10 specific siRNA and non-targeting scrambled siRNA for $48 \mathrm{~h}$. Subsequently, invasion assays were assessed. ADAM10 specific siRNA transfection clearly reduced the invasiveness of pancreatic cancer cells as compared to the control (Fig. 3C). In order to examine the effects of ADAM10 on pancreatic cancer cell migration an in vitro wound healing assay was performed. Migration of ASPC-1 and COLO-357 cells was markedly reduced after ADAM10 siRNA transfection compared to control. COLO-357 showed only a limited overall migration capacity (Fig. 3D and E).

Effects of ADAM10 silencing on E-cadherin expression on $m R N A$ and protein. ADAM10 is the main sheddase of the cell adhesion molecule E-cadherin in the human keratinocyte cell line $\mathrm{HaCaT}$ (27). During the shedding process the full length E-cadherin $(120 \mathrm{kDa})$ is cleaved, generating a $38-\mathrm{kDa}$ C-terminal fragment (CTF1) which can be consecutively processed to a 33-kDa CTF2 (27). We performed qRT-PCR and Western blot analysis to evaluate effects of ADAM10 gene silencing on E-cadherin expression and processing in ASPC- 1 and COLO- 357 cells. To exclude effects of ADAM10 silencing on E-cadherin mRNA expression, qRT-PCR was performed. On an mRNA level no difference in E-cadherin expression compared to controls was detectable after ADAM10 silencing (Fig. 4A). Similar results were found at the protein levels. No reduction of CTF1 or accumulations of the full length protein (FL) after ADAM10 siRNA were detectable (Fig. 4B).

\section{Discussion}

ADAMs are membrane-associated metalloproteinases with a complex multidomain structure and various functions. They have for example the potential to regulate the remodeling of extracellular matrix proteins and to influence cell migration $(46,47)$. There are many examples for expression and upregulation of proteolytic ADAMs in tumor tissues and cancer cell lines $(16,48)$. In pancreatic cancer, ADAMs, e.g. ADAM8, ADAM9, ADAM15, and ADAM17 may be upregulated and correlate to invasiveness and aggressiveness of the tumor $(2,3,35)$.

The role of the ADAM family member ADAM10 in pancreatic cancer has not been determined. In the present study analysis of ADAM10 mRNA levels in tissues showed no relevant differences between NP, CP and PDAC. However, ADAM10 in NP and CP showed a narrow range of mRNA levels, whereas in PDAC a wider range of mRNA expression levels was observed. The wide range in cancer tissues might be explained by different expression patterns of the tumor cells and further explained by the heterogeneous composition of the microenvironment of PDAC tissues, e.g. infiltrating immune cells or desmoplastic stroma. In contrast to these results Ko et al demonstrated an overexpression of ADAM10 on an mRNA level in oral squamous cell carcinoma (28). Additionally we detected in all analyzed PDAC cell lines ADAM10 mRNA.

On the protein level in CP and PDAC ADAM10 was strongly expressed in tubular complexes representing a potential precancerous lesion in inflammatory altered pancreatic tissue as well as in cancer cells. Here it was mainly localized in the cytoplasm, and in some cells additionally in the nucleus, as evaluated by immunohistochemistry. In NP tissue, ducts showed only focal immunoreactivity for ADAM10, whereas moderate diffuse staining of acinar cells and islets were observed. The results correlate with McCulloch et al for prostate cancer in which tumor cells also demonstrated nuclear and perinuclear immunoreactivity, respectively, without membrane staining (18), supporting their hypothesis 
that ADAM10 is responsible not only for extracellular but also for intracellular processes in tumor cells especially by interacting with nuclear proteins or DNA. Immunoblotting demonstrated an expression of the inactive proform $(\sim 100 \mathrm{kDa})$ as well as of the active form $(\sim 60 \mathrm{kDa})$ of ADAM10. Interestingly, in cell lines the inactive proform of ADAM10 was predominantly expressed whereas in tissue samples a stronger expression of the active form was detectable. Besides the heterogeneous composition of tissue samples, a possible explanation for the predominance of the active form in tissues might be an implementation of pathways induced in an autocrine manner by the tumor cells themselves or by the peritumoral microenvironment resulting in activation of ADAM10 in PDAC cells. It has been reported that e.g. the ADAM10 activator furin shows an increased expression in breast (49), and ovarian cancer (50) and head and neck squamous cell carcinoma (51). Concerning the discrepancy between mRNA and protein expression of tested PDAC cell lines, one may speculate about an effect of different mRNA splice-variants or mRNA stability as well as posttranscriptional modification or variable protein stability (52). Pro-proliferative effects of ADAM10 by modulating $\beta$-catenin signaling through E-cadherin shedding and increasing gene cyclin D1 levels have been described by Shtutman et al (42). In contrast to these results we could not find a direct or indirect effect of ADAM10 on cell proliferation of PDAC cells after stimulation with rhADAM10 or after silencing with siRNA transfection. Cell-cell and cell-matrix interactions play a pivotal role in the process of tumor cell invasion and dissemination. ADAMs are crucial factors in liberating migration promoting molecules such as chemokines (23), cytokines (20), and their receptors (53) and moreover in interacting with cell adhesion molecules $(30,54)$. ADAM10 was identified in recent studies as a major sheddase of adhesion molecules such as E-cadherin (27) and CXCL16 (23) in its membrane-bound form. Wente et al have shown that incubation of cancer cells with CXCL16 significantly increased invasiveness of PDAC cells (55).

Furthermore, ADAM10 siRNA transfected PDAC cells, liberating less soluble CXCL16, are responsible for a significant decrease of the migration of neutrophil granulocytes to the tumor (56). These findings corroborate the role of ADAM10 as a main source of cytokine liberation supporting tumor cell migration (14). Considering the fact of ADAM10 being a main sheddase of CXCL16 these findings suggest an involvement of ADAM10 not only in PDAC cell invasion but also in the interaction between tumor and immune cells. To gain more information about the influence of ADAM10 on PDAC cell invasion an invasion assay was performed. Indeed, ADAM10 silencing suppressed the invasiveness of PDAC cells. Furthermore, ADAM10-mediated release is reported to enhance tumor cell dissemination by increasing migration of ovarian and uterine carcinoma cells (29). To evaluate the role of ADAM10 in PDAC cell migration, we tested ADAM10 siRNA transfected cells in a wound healing assay. Migration in transfected cell lines was markedly reduced compared to controls. The reduced migration might be a result of less ADAM10-mediated shedding of cell adhesion molecules such as cadherins as well as chemotactic cytokines e.g. CXCL16. Consistent with our results Maretzky et al found an enhanced motility in ADAM10-transfected HaCaT cells (27). To elucidate the role of ADAM10 in E-cadherin shedding in PDAC cells, Western blot analysis with antibodies detecting the C-terminal fragment 1 (CTF1) and the full length (FL) E-cadherin were performed after ADAM10 silencing. While Maretzky et al demonstrated a distinct reduction of CTF1 generation and an accumulation of the FL protein by immunoblot analyses, processing of E-cadherin after ADAM10 silencing in PDAC cells was unaltered. This suggests that in PDAC other substrates of ADAM10, influencing adhesion or migration, are processed predominantly.

In conclusion, at the protein level a trend toward upregulation of ADAM10 in PDAC was observed. ADAM10 promotes cancer cell migration and invasion of PDAC cells, suggesting a crucial role of ADAM10 in tumor cell invasion and in metastasis of pancreatic cancer.

\section{Acknowledgements}

We thank Thomas Giese, Institute for Immunology, University of Heidelberg, for performing the RT-PCR and Klaus Felix, Department of Surgery, University of Heidelberg for continuous technical support and intensive discussion of results.

\section{References}

1. Jemal A, Siegel R, Ward E, Hao Y, Xu J and Thun MJ: Cancer statistics, 2009. CA Cancer J Clin 59: 225-249, 2009.

2. Ringel J, Jesnowski R, Moniaux N, et al: Aberrant expression of a disintegrin and metalloproteinase $17 /$ tumor necrosis factor-alpha converting enzyme increases the malignant potential in human pancreatic ductal adenocarcinoma. Cancer Res 66: 9045-9053, 2006.

3. Valkovskaya N, Kayed H, Felix K, et al: ADAM8 expression is associated with increased invasiveness and reduced patient survival in pancreatic cancer. J Cell Mol Med 11: 1162-1174, 2007.

4. Gould RJ, Polokoff MA, Friedman PA, Huang TF, Holt JC, Cook JJ and Niewiarowski S: Disintegrins: a family of integrin inhibitory proteins from viper venoms. Proc Soc Exp Biol Med 195: 168-171, 1990.

5. Kheradmand F and Werb Z: Shedding light on sheddases: role in growth and development. Bioessays 24: 8-12, 2002.

6. Blobel CP: ADAMs: key components in EGFR signalling and development. Nat Rev Mol Cell Biol 6: 32-43, 2005.

7. Seals DF and Courtneidge SA: The ADAMs family of metalloproteases: multidomain proteins with multiple functions. Genes Dev 17: 7-30, 2003.

8. Yuan R, Primakoff P and Myles DG: A role for the disintegrin domain of cyritestin, a sperm surface protein belonging to the ADAM family, in mouse sperm-egg plasma membrane adhesion and fusion. J Cell Biol 137: 105-112, 1997.

9. Eto K, Huet C, Tarui T, et al: Functional classification of ADAMs based on a conserved motif for binding to integrin alpha 9beta 1: implications for sperm-egg binding and other cell interactions. J Biol Chem 277: 17804-17810, 2002.

10. Gilpin BJ, Loechel F, Mattei MG, Engvall E, Albrechtsen R and Wewer UM: A novel, secreted form of human ADAM 12 (meltrin alpha) provokes myogenesis in vivo. J Biol Chem 273: 157-166, 1998 .

11. Kawaguchi N, Xu X, Tajima R, et al: ADAM 12 protease induces adipogenesis in transgenic mice. Am J Pathol 160: 1895-1903, 2002

12. Masaki M, Kurisaki T, Shirakawa K and Sehara-Fujisawa A: Role of meltrin alpha (ADAM12) in obesity induced by high-fat diet. Endocrinology 146: 1752-1763, 2005.

13. Yang P, Baker KA and Hagg T: The ADAMs family: coordinators of nervous system development, plasticity and repair. Prog Neurobiol 79: 73-94, 2006.

14. Garton KJ, Gough PJ and Raines EW: Emerging roles for ectodomain shedding in the regulation of inflammatory responses. J Leukoc Biol 79: 1105-1116, 2006. 
15. Holgate ST, Yang Y, Haitchi HM, et al: The genetics of asthma: ADAM33 as an example of a susceptibility gene. Proc Am Thorac Soc 3: 440-443, 2006.

16. Mochizuki S and Okada Y: ADAMs in cancer cell proliferation and progression. Cancer Sci 98: 621-628, 2007.

17. Chantry A, Gregson NA and Glynn P: A novel metalloproteinase associated with brain myelin membranes. Isolation and characterization. J Biol Chem 264: 21603-21607, 1989.

18. McCulloch DR, Akl P, Samaratunga H, Herington AC and Odorico DM: Expression of the disintegrin metalloprotease ADAM-10, in prostate cancer and its regulation by dihydrotestosterone, insulin-like growth factor I, and epidermal growth factor in the prostate cancer cell model LNCaP. Clin Cancer Res 10: 314-323, 2004

19. Black RA, Rauch CT, Kozlosky CJ, et al: A metalloproteinase disintegrin that releases tumour-necrosis factor-alpha from cells. Nature 385: 729-733, 1997

20. Lunn CA, Fan X, Dalie B, Miller K, Zavodny PJ, Narula SK and Lundell D: Purification of ADAM 10 from bovine spleen as a TNFalpha convertase. FEBS Lett 400: 333-335, 1997.

21. Hundhausen C, Misztela D, Berkhout TA, et al: The disintegrinlike metalloproteinase ADAM10 is involved in constitutive cleavage of CX3CL1 (fractalkine) and regulates CX3CL1mediated cell-cell adhesion. Blood 102: 1186-1195, 2003.

22. Schulte A, Schulz B, Andrzejewski MG, et al: Sequential processing of the transmembrane chemokines CX3CL1 and CXCL16 by alpha- and gamma-secretases. Biochem Biophys Res Commun 358: 233-240, 2007.

23. Abel S, Hundhausen C, Mentlein R, et al: The transmembrane CXC-chemokine ligand 16 is induced by IFN-gamma and TNF-alpha and shed by the activity of the disintegrin-like metalloproteinase ADAM10. J Immunol 172: 6362-6372, 2004.

24. Gough PJ, Garton KJ, Wille PT, Rychlewski M, Dempsey PJ and Raines EW: A disintegrin and metalloproteinase 10mediated cleavage and shedding regulates the cell surface expression of CXC chemokine ligand 16. J Immunol 172: 3678-3685, 2004.

25. Gutwein P, Mechtersheimer S, Riedle S, et al: ADAM10mediated cleavage of L1 adhesion molecule at the cell surface and in released membrane vesicles. FASEB J 17: 292-294, 2003.

26. Uemura K, Kihara T, Kuzuya A, et al: Characterization of sequential N-cadherin cleavage by ADAM10 and PS1. Neurosci Lett 402: 278-283, 2006.

27. Maretzky T, Reiss K, Ludwig A, et al: ADAM10 mediates E-cadherin shedding and regulates epithelial cell-cell adhesion, migration, and beta-catenin translocation. Proc Natl Acad Sci USA 102: 9182-9187, 2005

28. Ko SY, Lin SC, Wong YK, Liu CJ, Chang KW and Liu TY: Increase of disintergin metalloprotease 10 (ADAM10) expression in oral squamous cell carcinoma. Cancer Lett 245: 33-43, 2007.

29. Fogel M, Gutwein P, Mechtersheimer S, et al: L1 expression as a predictor of progression and survival in patients with uterine and ovarian carcinomas. Lancet 362: 869-875, 2003.

30. Gavert N, Conacci-Sorrell M, Gast D, Schneider A, Altevogt P, Brabletz T and Ben-Ze'ev A: L1, a novel target of beta-catenin signaling, transforms cells and is expressed at the invasive front of colon cancers. J Cell Biol 168: 633-642, 2005.

31. Otto T, Rembrink K, Goepel M, Meyer-Schwickerath M and Rubben H: E-cadherin: a marker for differentiation and invasiveness in prostatic carcinoma. Urol Res 21: 359-362, 1993.

32. Yonemura Y, Ninomiya I, Kaji M, et al: Decreased E-cadherin expression correlates with poor survival in patients with gastric cancer. Anal Cell Pathol 8: 177-190, 1995.

33. Ryniers F, Stove C, Goethals M, et al: Plasmin produces an E-cadherin fragment that stimulates cancer cell invasion. Biol Chem 383: 159-165, 2002.

34. Grutzmann R, Luttges J, Sipos B, et al: ADAM9 expression in pancreatic cancer is associated with tumour type and is a prognostic factor in ductal adenocarcinoma. Br J Cancer 90: 1053-1058, 2004.

35. Yamada D, Ohuchida K, Mizumoto K, et al: Increased expression of ADAM 9 and ADAM 15 mRNA in pancreatic cancer. Anticancer Res 27: 793-799, 2007.

36. Erkan M, Kleeff J, Esposito I, et al: Loss of BNIP3 expression is a late event in pancreatic cancer contributing to chemoresistance and worsened prognosis. Oncogene 24: 4421-4432, 2005.
37. Tousseyn T, Thathiah A, Jorissen E, et al: ADAM10, the ratelimiting protease of regulated intramembrane proteolysis of Notch and other proteins, is processed by ADAMS-9, ADAMS-15, and the gamma-secretase. J Biol Chem 284: 11738-11747, 2009.

38. Prenzel N, Zwick E, Daub H, Leserer M, Abraham R, Wallasch C and Ullrich A: EGF receptor transactivation by G-protein-coupled receptors requires metalloproteinase cleavage of proHB-EGF. Nature 402: 884-888, 1999.

39. Yan Y, Shirakabe K and Werb Z: The metalloprotease Kuzbanian (ADAM10) mediates the transactivation of EGF receptor by G protein-coupled receptors. J Cell Biol 158: 221-226, 2002.

40. Hinkle CL, Mohan MJ, Lin P, Yeung N, Rasmussen F, Milla ME and Moss ML: Multiple metalloproteinases process protransforming growth factor-alpha (proTGF-alpha). Biochemistry 42: 2127-2136, 2003

41. Sahin U, Weskamp G, Kelly K, et al: Distinct roles for ADAM10 and ADAM17 in ectodomain shedding of six EGFR ligands. J Cell Biol 164: 769-779, 2004.

42. Shtutman M, Zhurinsky J, Simcha I, Albanese C, D'Amico M, Pestell R and Ben-Ze'ev A: The cyclin D1 gene is a target of the beta-catenin/LEF-1 pathway. Proc Natl Acad Sci USA 96: 5522-5527, 1999 .

43. Schramme A, Abdel-Bakky MS, Kampfer-Kolb N, Pfeilschifter J and Gutwein P: The role of CXCL16 and its processing metalloproteinases ADAM10 and ADAM17 in the proliferation and migration of human mesangial cells. Biochem Biophys Res Commun 370: 311-316, 2008.

44. Hotary K, Allen E, Punturieri A, Yana I and Weiss SJ: Regulation of cell invasion and morphogenesis in a three-dimensional type I collagen matrix by membrane-type matrix metalloproteinases 1 , 2, and 3. J Cell Biol 149: 1309-1323, 2000.

45. Hotary KB, Allen ED, Brooks PC, Datta NS, Long MW and Weiss SJ: Membrane type I matrix metalloproteinase usurps tumor growth control imposed by the three-dimensional extracellular matrix. Cell 114: 33-45, 2003.

46. Wolfsberg TG, Primakoff P, Myles DG and White JM: ADAM, a novel family of membrane proteins containing A Disintegrin and metalloprotease domain: multipotential functions in cellcell and cell-matrix interactions. J Cell Biol 131: 275-278, 1995.

47. Black RA and White JM: ADAMs: focus on the protease domain. Curr Opin Cell Biol 10: 654-659, 1998.

48. Rocks N, Paulissen G, El Hour M, et al: Emerging roles of ADAM and ADAMTS metalloproteinases in cancer. Biochimie 90: 369-379, 2008.

49. Cheng M, Watson PH, Paterson JA, Seidah N, Chretien M and Shiu RP: Pro-protein convertase gene expression in human breast cancer. Int J Cancer 71: 966-971, 1997.

50. Page RE, Klein-Szanto AJ, Litwin S, et al: Increased expression of the pro-protein convertase furin predicts decreased survival in ovarian cancer. Cell Oncol 29: 289-299, 2007.

51. Bassi DE, Mahloogi H, Al-Saleem L, Lopez De CR, Ridge JA and Klein-Szanto AJ: Elevated furin expression in aggressive human head and neck tumors and tumor cell lines. Mol Carcinog 31: 224-232, 2001.

52. Zhou DC, Zittoun R and Marie JP: Expression of multidrug resistance-associated protein (MRP) and multidrug resistance (MDR1) genes in acute myeloid leukemia. Leukemia 9: 1661-1666, 1995 .

53. Matthews V, Schuster B, Schutze S, et al: Cellular cholesterol depletion triggers shedding of the human interleukin-6 receptor by ADAM10 and ADAM17 (TACE). J Biol Chem 278: 38829-38839, 2003.

54. Maretzky T, Schulte M, Ludwig A, et al: L1 is sequentially processed by two differently activated metalloproteases and presenilin/gamma-secretase and regulates neural cell adhesion, cell migration, and neurite outgrowth. Mol Cell Biol 25: 9040-9053, 2005.

55. Wente MN, Gaida MM, Mayer C, et al: Expression and potential function of the CXC chemokine CXCL16 in pancreatic ductal adenocarcinoma. Int J Oncol 33: 297-308, 2008.

56. Gaida MM, Gunther F, Wagner C, et al: Expression of the CXCR6 on polymorphonuclear neutrophils in pancreatic carcinoma and in acute, localized bacterial infections. Clin Exp Immunol 154: 216-223, 2008. 